\title{
Comparative studies on phytochemistry, antibacterial and antifungal properties of Alstonia scholaris and Millettia pinnata
}

\author{
Sobiya Shafique • Waheed Akram • Tehmina Anjum • \\ Aqeel Ahmad • Shazia Shafique
}

Received: 22 February 2013 / Accepted: 21 March 2014 /Published online: 8 April 2014

(C) Australasian Plant Pathology Society Inc. 2014

\begin{abstract}
Phytochemical profiles and antimicrobial activities of $A$. scholaris and M. pinnata extracts have been assessed. Test microorganisms were evaluated for their susceptibility against aqueous, methanol, ethanol, $\mathrm{n}$-hexane and chloroform extracts from leaves of both plant species. Antibacterial effects were evaluated against eight bacterial species (three gram positive and five gram negative) of high economic importance, whereas antifungal effects were evaluated against three devastating fungal pathogens. Maximum number of phytochemical groups was noted in methanol and aqueous extracts of both plant species which showed the best antibacterial activities. This indicates that methanol extract contained a wide range of antibiotic compounds, whereas ethanol extract was least effective against tested bacterial and fungal species. This study supports the use of plant origin pesticides against pathogenic microbes.
\end{abstract}

Keywords Antimycotic · Phytochemistry $\cdot$ Allelochemicals · Antibiotics

Plants have long been used as a source of antibiotics but the potential of plants as sources for new drugs is still generally

\author{
S. Shafique $\cdot$ W. Akram $\cdot$ T. Anjum $\cdot$ A. Ahmad $(\varangle) \cdot$ S. Shafique \\ Institute of Agricultural Sciences, University of the Punjab, Lahore, \\ Pakistan \\ e-mail: aqeelahmad1@gmail.com \\ S. Shafique \\ e-mail: drsobi81@gmail.com \\ W. Akram \\ e-mail: meher_waheed@yahoo.com \\ T. Anjum \\ e-mail: tehminaanjum@yahoo.com \\ S. Shafique \\ e-mail: drshazi81@gmail.com
}

unexplored. Plant originated drugs also have the advantage of being less hazardous to environmental health (Bajwa et al. 2008; Shafique et al. 2011). Thus, the plant kingdom is being screened for new and effective chemotherapeutic agents (Shafique et al. 2013). Despite all efforts made by researchers, only a few plant species have been phytochemically investigated until now (Sabir et al. 2007). Plant extracts of many higher plants have been reported to exhibit antibacterial, antifungal and insecticidal properties under laboratory trials (Akram et al. 2013; Bouamama et al. 2006). Among these plants, Alstonia scholaris is an evergreen tropical tree having latex rich in alkaloids (Hussain et al. 2010).

Another medicinally important plant, Millettia pinnata (Fabaceae), contains oils and juices with strong antiseptic and antimicrobial properties (Baswa et al. 2001). Considering plants as great sources of antibiotic agents, an investigation was performed to study the local flora for comparative phytochemistry, antibacterial and antifungal activity.

Leaves of Alstonia and Milletia plant species were collected from University of the Punjab, Lahore, Pakistan $\left(31^{\circ} 17^{\prime} 34^{\prime \prime}\right.$ N $74^{\circ} 10^{\prime} 29^{\prime \prime} \mathrm{E}$ ), in December 2012. Leaves of both plants were washed, dried and blended into fine powder separately. Aqueous, methanol, ethanol, n-hexane and chloroform extracts were prepared using the method of Azu and Onyeagba (2007). Extraction of plant biochemicals was performed at room temperature $\left(25 \pm 2{ }^{\circ} \mathrm{C}\right)$ using solvents for plant biochemicals. Solvents of all extracts were removed using vacuum drier at $40{ }^{\circ} \mathrm{C}$.

Different extracts were phytochemically analysed using the method of Brain and Turner (1975). Briefly, Mayer's reagent was used for alkaloids analysis; magnesium and acetone solutions were used for anthraquinones test; concentrated $\mathrm{HCl}$ and magnesium solutions were used to determine flavonoids; ferric chloride was used for phenols; thionyl chloride crystals were used in terpenoids test; lead acetate was used for 
Table 1 Observations of phytochemical screening

Aqu Aqueous, Eth Ethanol, Meth Methanol, $n$-Hex n-Hexane, Chlo Chloroform

\begin{tabular}{|c|c|c|c|c|c|c|c|c|c|c|}
\hline \multirow[t]{2}{*}{ Phytochemical } & \multicolumn{5}{|c|}{ Alstonia scholaris } & \multicolumn{5}{|c|}{ Millettia pinnata } \\
\hline & Aqu. & Eth. & Meth. & n-Hex. & Chlo. & Aqu. & Eth. & Meth. & n-Hex. & Chlo. \\
\hline Anthraquinone & + & - & - & - & + & + & - & + & - & - \\
\hline Alkaloids & + & + & + & - & - & + & + & + & - & - \\
\hline Flavonoids & - & - & + & + & - & - & - & + & + & + \\
\hline Phenolics & - & + & + & - & + & + & + & + & + & + \\
\hline Saponins & - & - & - & - & + & - & + & + & + & - \\
\hline Steroids & + & - & + & + & - & - & - & - & + & - \\
\hline Tannins & + & + & + & + & - & + & - & + & - & \\
\hline Terpenoids & - & - & - & + & + & - & - & + & - & + \\
\hline
\end{tabular}

determination of tannins and water was used to perceive the presence of saponins and formation of foam.

Bacterial cultures of Bacillus subtilis (FCBP-0174), Bacillus fortis (FCBP-0162), Staphylococcus aureus (FBT0014), Escherichia coli (FCBP-0257), Pseudomonas syringe (FCBP-0275), Pseudomonas putida (FBT-0032), Erwinia amylovora (FBT-0053), Burkholderia gladioli (FBT-0034) and fungal cultures of Alternaria alternata (FCBP-0003), Alternaria solani (FCBP-0831) and Fusarium oxysporum (FCBP-0866) were obtained from the Culture Conservatory of the Institute of Agricultural Sciences, University of the
Punjab, Lahore Pakistan. Bacteria were cultured upon nutrient broth (NB) at $37{ }^{\circ} \mathrm{C}$ and fungal cultures were maintained on potato dextrose agar (PDA) at $28^{\circ} \mathrm{C}$.

Bacterial inoculum $\left(10^{6}\right.$ cells $\left./ \mathrm{mL}\right)$ was prepared by recording its absorbance at $550 \mathrm{~nm}$ (Sutton 2011) and fungal inoculum of $10^{6}$ spores $/ \mathrm{mL}$ was also prepared using a haemocytometer (Valgas et al. 2007). Both of the inocula were used in antibacterial and antifungal assays performed by disc diffusion method. Filter paper discs $(0.5 \mathrm{~cm}$ diameter) were impregnated with $100 \mu \mathrm{g}$ of 300,200 and $100 \mu \mathrm{g} / \mathrm{mL}$ concentrations of extracts in a way that each disc contained 30, 20

Table 2 Antibacterial activity of extracts of A. scholaris

\begin{tabular}{|c|c|c|c|c|c|c|c|c|c|}
\hline \multirow[t]{3}{*}{ Extracts } & \multirow[t]{3}{*}{ Conc. in $\mu \mathrm{g} / \mathrm{disc}$} & \multicolumn{8}{|c|}{ Zone of inhibition in $\mathrm{mm}$} \\
\hline & & \multicolumn{3}{|c|}{ Gram positive } & \multicolumn{5}{|c|}{ Gram negative } \\
\hline & & B. subtilis & B. fortis & S. aureus & E. coli & P. syringae & P. putida & E. amylovora & B. gladioli \\
\hline \multirow[t]{3}{*}{ Aqueous } & 10 & $4 \pm 0.81$ & $-\mathrm{j}$ & $15 \pm 0.5 \mathrm{c}$ & -1 & $11 \pm 1.8 \mathrm{~g}$ & $-\mathrm{j}$ & $5 \pm 0.9 \mathrm{fg}$ & $7 \pm 0.4 \mathrm{~d}$ \\
\hline & 20 & $7 \pm 0.6 \mathrm{j}$ & $23 \pm 0.7 \mathrm{~d}$ & $26 \pm 0.9 b$ & $18 \pm 0.8 \mathrm{c}$ & $20 \pm 0.6 \mathrm{~d}$ & $7 \pm 0.6 \mathrm{i}$ & $11 \pm 0.3 \mathrm{c}$ & $11 \pm 0.9 \mathrm{c}$ \\
\hline & 30 & $13 \pm 0.3 \mathrm{~g}$ & $31 \pm 0.9 \mathrm{~b}$ & $34 \pm 1.6 \mathrm{a}$ & $23 \pm 0.6 b$ & $26 \pm 0.3 \mathrm{c}$ & $11 \pm 1.3 \mathrm{fg}$ & $14 \pm 0.4 b$ & $18 \pm 1.4 \mathrm{~b}$ \\
\hline \multirow[t]{3}{*}{ Ethanol } & 10 & $5 \pm 0.1 \mathrm{k}$ & $-\mathrm{j}$ & $-\mathrm{i}$ & -1 & $-\mathrm{k}$ & $5 \pm 0.1$ & $-\mathrm{j}$ & $-\mathrm{h}$ \\
\hline & 20 & $9 \pm 0.2 \mathrm{i}$ & $10 \pm 0.5 \mathrm{~h}$ & $-\mathrm{i}$ & -1 & $8 \pm 0.2 \mathrm{~h}$ & $6 \pm 0.1$ & $3 \pm 0.4 \mathrm{~h}$ & $-\mathrm{h}$ \\
\hline & 30 & $13 \pm 0.5 \mathrm{~g}$ & $16 \pm 0.4 f$ & $5 \pm 1.2 \mathrm{~g}$ & $4 \pm 0.3 \mathrm{k}$ & $13 \pm 0.2 \mathrm{~g}$ & $10 \pm 0.2 \mathrm{~g}$ & $4 \pm 0.1 \mathrm{~g}$ & $2 \pm 0.3 \mathrm{~g}$ \\
\hline \multirow[t]{3}{*}{ Methanol } & 10 & $4 \pm 0.11$ & $7 \pm 0.7 \mathrm{i}$ & $6 \pm 0.2 \mathrm{~g}$ & $4 \pm 0.2 \mathrm{k}$ & $8 \pm 0.1 \mathrm{~h}$ & $-\mathrm{j}$ & $-\mathrm{j}$ & $2 \pm 0.1 \mathrm{~g}$ \\
\hline & 20 & $9 \pm 0.3 \mathrm{i}$ & $12 \pm 0.4 \mathrm{~g}$ & $9 \pm 0.1 \mathrm{e}$ & $6 \pm 0.1 \mathrm{j}$ & $14 \pm 0.2 \mathrm{f}$ & $11 \pm 0.3 f$ & $9 \pm 1.2 \mathrm{~d}$ & $3 \pm 0.2 \mathrm{f}$ \\
\hline & 30 & $15 \pm 0.3 \mathrm{f}$ & $19 \pm 1.5 \mathrm{e}$ & $11 \pm 0.6 \mathrm{~d}$ & $9 \pm 0.1 \mathrm{~h}$ & $18 \pm 0.3 \mathrm{e}$ & $19 \pm 1.6 \mathrm{c}$ & $14 \pm 0.5 b$ & $7 \pm 0.2 \mathrm{~d}$ \\
\hline \multirow[t]{3}{*}{ n-Hexane } & 10 & $-\mathrm{m}$ & $13 \pm 0.7 \mathrm{~g}$ & $-\mathrm{i}$ & $11 \pm 0.4 \mathrm{~g}$ & $-\mathrm{k}$ & $9 \pm 0.5 \mathrm{~h}$ & $2 \pm 0.1 \mathrm{i}$ & $-\mathrm{h}$ \\
\hline & 20 & $16 \pm 0.1 \mathrm{e}$ & $19 \pm 0.9 \mathrm{e}$ & $3 \pm 0.3 \mathrm{~h}$ & $14 \pm 0.3 \mathrm{e}$ & $4 \pm 0.1 \mathrm{j}$ & $13 \pm 0.4 \mathrm{e}$ & $3 \pm 0.5 \mathrm{~h}$ & $-\mathrm{h}$ \\
\hline & 30 & $22 \pm 0.5 \mathrm{c}$ & $25 \pm 1.4 \mathrm{~d}$ & $7 \pm 0.7 f$ & $19 \pm 0.3 \mathrm{c}$ & $7 \pm 0.2 \mathrm{i}$ & $17 \pm 0.2 \mathrm{~d}$ & $6 \pm 0.3 f$ & $3 \pm 0.3 \mathrm{f}$ \\
\hline \multirow[t]{3}{*}{ Chloroform } & 10 & $10 \pm 0.2 \mathrm{~h}$ & $-\mathrm{j}$ & $3 \pm 0.3 \mathrm{~h}$ & $8 \pm 0.1 \mathrm{i}$ & $-\mathrm{k}$ & $13 \pm 1.2 \mathrm{e}$ & - & $4 \pm 0.1 \mathrm{e}$ \\
\hline & 20 & $18 \pm 0.4 \mathrm{~d}$ & $18 \pm 0.8 \mathrm{e}$ & $7 \pm 0.2 f$ & $13 \pm 0.5 \mathrm{f}$ & $-\mathrm{k}$ & $20 \pm 1.5 \mathrm{c}$ & $7 \pm 0.3 e$ & $9 \pm 1.3 \mathrm{c}$ \\
\hline & 30 & $26 \pm 1.5 b$ & $28 \pm 1.4 \mathrm{c}$ & $11 \pm 0.5 \mathrm{~d}$ & $16 \pm 0.4 \mathrm{~d}$ & $31 \pm 1.2 b$ & $27 \pm 0.7 b$ & $13 \pm 1.2 b$ & $16 \pm 1.0 \mathrm{~b}$ \\
\hline Nystatin & 10 & $29 \pm 0.2 \mathrm{a}$ & $34 \pm 0.2 \mathrm{a}$ & $35 \pm 1.2 \mathrm{a}$ & $27 \pm 0.5 \mathrm{a}$ & $33 \pm 0.1 \mathrm{a}$ & $30 \pm 0.8 \mathrm{a}$ & $28 \pm 0.4 \mathrm{a}$ & $26 \pm 0.5 \mathrm{a}$ \\
\hline
\end{tabular}

Values were expressed as Mean \pm Standard Error (S.E). Data were statistically analysed through Duncan's Multiple Range Test (DMRT) at $p \leq 0.05$. DMRT was performed using statistical package DSAASTAT (Onofri, Italy) and alphabetic letters showing significance of data follow each value. Values followed by similar letters showed no significant difference. Values followed by single consecutive letters showed significant difference; whereas, values followed by more than one letter showed no significant difference 
and $10 \mu \mathrm{g}$ extract, respectively. Discs impregnated with DMSO and Nystatin $100 \mu \mathrm{g}$ were used as negative and positive controls, respectively. Activity was determined after 5 days of incubation.

Eight phytochemical classes (i.e. alkaloids, flavonoids, phenolics, steroids, tannins anthraquinone, saponins and terpenoids) were extracted using methanol as extraction solvent. Four different classes of biochemicals were observed in ethanol extract (Table 1), whereas five different phytochemical classes were observed in aqueous extract. Moreover, chloroform extract showed the presence of anthraquinones, flavonoids, phenolics, saponins and terpenoids. Presence of flavonoids, phenolics, saponins, steroids, tannins and terpenoides were observed in $\mathrm{n}$-hexane extract (Table 1).

Maximum number of phytochemicals was observed in the methanol extract of $M$. pinnata; only steroids being absent. Aqueous extract contained anthraquinones, alkaloids, phenolics and tannins. Minimum number of phytochemical classes was observed in ethanol and chloroform extracts (Table 1).

All of the extracts presented varying antibacterial activity for tested bacterial strains at different concentrations. Leaf extracts of $A$. scholaris had antibacterial effects against different bacterial strains (Table 2). Methanol extract of $A$. scholaris presented the highest antibacterial activity. Methanol extract was able to inhibit growth of the most bacterial strains even at the lowest concentration of $100 \mu \mathrm{g} / \mathrm{mL}$. However, P. putida and $E$. amylovora were found resistant at the lower concentration of methanol extract. Ethanol extract was least effective against bacterial strains of both gram positive and gram negative types. Even at a concentration of $200 \mu \mathrm{g} / \mathrm{mL}, S$. aureus, E. coli and B. gladioli were resistant against ethanol extract of A. scholaris. Maximum inhibitory zone diameter $(34 \mathrm{~mm})$ was observed in the aqueous extract of $A$. scholaris against $S$. aureus at the concentration of $300 \mu \mathrm{g} / \mathrm{mL}$. The same bacterial strain was found to be resistant against $n$-hexane extract at the concentration of $100 \mu \mathrm{g} / \mathrm{mL}$.

Methanol extract of M. pinnata was found effective against bacterial strains of both gram negative and gram positive types (Table 3). Three bacterial strains (i.e. E. coli, P. putida and $B$. gladioli) were found to be resistant against lower concentrations of ethanol extract. One bacterial species, E. coli, was found to be resistant against $\mathrm{n}$-hexane extract at the concentration of 100 and $200 \mu \mathrm{g} / \mathrm{mL}$. Bacterial species, P. putida, was also found resistant against chloroform extract of M. pinnata at lower concentrations but susceptible at higher concentrations. All other extracts represented average antibacterial activity at different concentrations.

Appreciable antifungal activity was observed in methanol extract of M. pinnata in which all fungal pathogens showed significant zones of inhibition at $100 \mu \mathrm{g} / \mathrm{mL}$ concentration.

Table 3 Antibacterial activity of extracts of $M$. pinnata

\begin{tabular}{|c|c|c|c|c|c|c|c|c|c|}
\hline \multirow[t]{3}{*}{ Extracts } & \multirow[t]{3}{*}{ Conc.in $\mu \mathrm{g} / \mathrm{disc}$} & \multicolumn{8}{|c|}{ Zone of inhibition (mm) } \\
\hline & & \multicolumn{3}{|c|}{ Gram positive } & \multicolumn{5}{|c|}{ Gram negative } \\
\hline & & B. subtilis & B. fortis & S. aureus & E. coli & P. syringae & P. putida & E. amylovora & B. gladioli \\
\hline \multirow[t]{3}{*}{ Aqueous } & 10 & $13 \pm 1.3 \mathrm{e}$ & $16 \pm 0.4 \mathrm{~d}$ & $-\mathrm{j}$ & $3 \pm 0.5 \mathrm{f}$ & $8 \pm 1.2 \mathrm{~g}$ & $15 \pm 0.4 \mathrm{~g}$ & $9 \pm 0.4 \mathrm{~g}$ & $12 \pm 0.4 \mathrm{f}$ \\
\hline & 20 & $21 \pm 0.4 \mathrm{c}$ & $22 \pm 1.2 \mathrm{c}$ & $4 \pm 0.4 \mathrm{i}$ & $8 \pm 0.4 \mathrm{~d}$ & $15 \pm 0.3 \mathrm{~d}$ & $17 \pm 0.2 \mathrm{e}$ & $16 \pm 0.1 \mathrm{e}$ & $18 \pm 0.6 \mathrm{~cd}$ \\
\hline & 30 & $32 \pm 1.7 \mathrm{~b}$ & $29 \pm 0.9 b$ & $7 \pm 1.3 \mathrm{~g}$ & $11 \pm 0.6 \mathrm{c}$ & $19 \pm 0.3 b$ & $23 \pm 0.8 \mathrm{bc}$ & $20 \pm 0.6 c$ & $21 \pm 1.0 \mathrm{~b}$ \\
\hline \multirow[t]{3}{*}{ Ethanol } & 10 & $-\mathrm{j}$ & $-\mathrm{k}$ & $5 \pm 0.4 \mathrm{~h}$ & $-\mathrm{g}$ & $5 \pm 1.3 \mathrm{~h}$ & $-\mathrm{j}$ & $4 \pm 0.9 \mathrm{j}$ & -1 \\
\hline & 20 & $-\mathrm{j}$ & $4 \pm 0.8 \mathrm{i}$ & $7 \pm 0.1 \mathrm{~g}$ & $3 \pm 0.4 \mathrm{f}$ & $11 \pm 0.7 \mathrm{e}$ & $14 \pm 0.5 \mathrm{~h}$ & $7 \pm 1.6 \mathrm{hi}$ & -1 \\
\hline & 30 & $3 \pm 0.4 \mathrm{i}$ & $10 \pm 0.2 \mathrm{f}$ & $16 \pm 0.3 \mathrm{e}$ & $7 \pm 0.6 \mathrm{de}$ & $16 \pm 0.3 \mathrm{c}$ & $20 \pm 0.5 \mathrm{~d}$ & $13 \pm 0.4 \mathrm{f}$ & $6 \pm 0.4 \mathrm{i}$ \\
\hline \multirow[t]{3}{*}{ Methanol } & 10 & $-\mathrm{j}$ & $2 \pm 1.0 \mathrm{j}$ & $7 \pm 0.8 \mathrm{~g}$ & $-\mathrm{g}$ & $10 \pm 1.4 \mathrm{f}$ & $11 \pm 0.5 \mathrm{i}$ & $8 \pm 0.2 \mathrm{~h}$ & $4 \pm 0.9 \mathrm{k}$ \\
\hline & 20 & $2 \pm 0.1$ & $6 \pm 0.3 \mathrm{~h}$ & $15 \pm 0.9 \mathrm{e}$ & $6 \pm 1.1 \mathrm{e}$ & $14 \pm 0.3 \mathrm{e}$ & $17 \pm 1.2 \mathrm{ef}$ & $17 \pm 0.4 \mathrm{~d}$ & $10 \pm 1.3 \mathrm{~g}$ \\
\hline & 30 & $7 \pm 1.1 \mathrm{~g}$ & $9 \pm 0.7 \mathrm{~g}$ & $19 \pm 1.3 \mathrm{~d}$ & $13 \pm 1.0 \mathrm{~b}$ & $19 \pm 0.7 b$ & $22 \pm 0.6 \mathrm{c}$ & $27 \pm 0.3 b$ & $17 \pm 0.5 \mathrm{~d}$ \\
\hline \multirow[t]{3}{*}{ n-Hexane } & 10 & $-\mathrm{j}$ & $6 \pm 0.4 \mathrm{~h}$ & $12 \pm 0.4 \mathrm{f}$ & $-\mathrm{g}$ & $8 \pm 0.3 \mathrm{~g}$ & $-\mathrm{j}$ & $6 \pm 0.1 \mathrm{i}$ & $5 \pm 0.3 \mathrm{j}$ \\
\hline & 20 & $5 \pm 0.5 \mathrm{~h}$ & $9 \pm 1.2 \mathrm{fgh}$ & $17 \pm 0.8 \mathrm{de}$ & $-\mathrm{g}$ & $11 \pm 0.4 \mathrm{f}$ & $16 \pm 0.1 \mathrm{f}$ & $9 \pm 0.6 \mathrm{~g}$ & $8 \pm 0.5 \mathrm{~h}$ \\
\hline & 30 & $7 \pm 0.2 \mathrm{~g}$ & $13 \pm 0.3 \mathrm{e}$ & $25 \pm 1.3 \mathrm{c}$ & $3 \pm 0.9 \mathrm{f}$ & $17 \pm 1.3 \mathrm{c}$ & $23 \pm 0.3 b$ & $13 \pm 0.5 f$ & $16 \pm 1.6 \mathrm{~d}$ \\
\hline \multirow[t]{3}{*}{ Chloroform } & 10 & $3 \pm 1.3 \mathrm{i}$ & $-\mathrm{k}$ & $7 \pm 1.2 \mathrm{~g}$ & $-\mathrm{g}$ & $3 \pm 0.4 \mathrm{i}$ & $-\mathrm{j}$ & $8 \pm 0.3 \mathrm{~h}$ & $8 \pm 0.3 \mathrm{~h}$ \\
\hline & 20 & $9 \pm 0.7 \mathrm{f}$ & $7 \pm 1.0 \mathrm{~h}$ & $18 \pm 1.4 \mathrm{~d}$ & $4 \pm 1.0 \mathrm{ef}$ & $6 \pm 0.7 \mathrm{~h}$ & $-\mathrm{j}$ & $12 \pm 1.2 \mathrm{f}$ & $13 \pm 0.2 \mathrm{e}$ \\
\hline & 30 & $17 \pm 0.6 \mathrm{~d}$ & $11 \pm 0.9 \mathrm{f}$ & $31 \pm 1.3 \mathrm{~b}$ & $10 \pm 0.7 \mathrm{c}$ & $10 \pm 1.0 \mathrm{f}$ & $22 \pm 0.9 \mathrm{bc}$ & $17 \pm 1.1 \mathrm{de}$ & $19 \pm 0.7 \mathrm{c}$ \\
\hline Nystatin & 10 & $35 \pm 0.4 \mathrm{a}$ & $34 \pm 0.5 \mathrm{a}$ & $36 \pm 1.2 \mathrm{a}$ & $26 \pm 0.7 \mathrm{a}$ & $34 \pm 1.1 \mathrm{a}$ & $24 \pm 0.1 \mathrm{a}$ & $31 \pm 0.2 \mathrm{a}$ & $23 \pm 0.1 \mathrm{a}$ \\
\hline
\end{tabular}

Values were expressed as Mean \pm Standard Error (S.E). Data were statistically analysed through DMRT at $p \leq 0.05$. Statistical package DSAASTAT (Onofri, Italy) for MS-Excel was used to perform the analysis and alphabetic letters following each value were used to mention significance of data. Values followed by similar letters showed no significant difference. Values followed by single consecutive letters showed significant difference; whereas, values followed by more than one letter showed no significant difference 
Radius of inhibition zone directly increased with the increased concentration of plant extract which clearly showed that fungal growth inhibition was strictly concentration dependent. It was closely followed by methanol extract of $A$. scholaris for which the minimum concentration also significantly inhibited fungal growth on synthetic medium (Table 4).

Aqueous extract of $M$. pinnata had the second highest antifungal activity. However, F. oxysporum showed resistance to that extract and a very little inhibition zone $(3 \mathrm{~mm})$ was observed at the highest concentration. However, both species of Alternaria were efficiently controlled by a lower concentration of that extract. Every adjacent higher concentration brought significant increase in antifungal activity. Zones of inhibition produced by aqueous extract of $M$. pinnatta against A. solani were 9, 16 and $27 \mathrm{~mm}$ at the concentrations of 100 , 200 and $300 \mu \mathrm{g} / \mathrm{mL}$, respectively. Two fungal species (F. oxysporum and $A$. solani) were resistant against aqueous extract of $A$. scholaris at $100 \mu \mathrm{g} / \mathrm{mL}$ concentration. However, at $200 \mu \mathrm{g} / \mathrm{mL}$ concentration, the extract controlled all three fungi efficiently.

Chloroform extract exhibited efficient antifungal activity at $200 \mu \mathrm{g} / \mathrm{mL}$. At the lowest concentration, most of the fungal species were resistant to the chloroform extract. At $100 \mu \mathrm{g} / \mathrm{mL}$ concentration of both plant species, $F$. oxysporum was resistant, while $A$. alternata and $A$. solani were resistant to least concentrations of $A$. scholaris and $M$. pinnata, respectively. Increased concentrations of these extracts significantly inhibited fungal growth up to $32 \mathrm{~mm}$ zone of inhibition in case of A.alternata with $300 \mu \mathrm{g} / \mathrm{mL}$ of $M$. pinnata extracts.

Antifungal potential was decreased from n-Hexane to ethanol extract. Only one fungal species was resistant to $200 \mu \mathrm{g} /$ $\mathrm{mL} \mathrm{n}-\mathrm{Hexane}$ plant extract but this number was doubled in $200 \mu \mathrm{g} / \mathrm{mL}$ of ethanol extract of the same plants. A concentration dependent trend was also found in both n-Hexane and ethanol extracts.

In the qualitative phytochemical studies, it was observed that secondary metabolites of leaves of both plant species were more soluble in methanol suggesting that methanol is a good solvent for recovery of most of the phytochemicals from plants. Among both types of plants, ethanol extract was least effective against both bacteria and fungi. This can be attributed to this solvent being unable to dissolve many types of phytochemical groups.

These phytochemical constituents are valuable sources of antimicrobial agents (Bashir et al. 2013). Scalbert (1991) and Aboaba et al. (2006) reported antimicrobial properties of tannins in their investigations. Other compounds such as alkaloids (Damintoti et al. 2005), saponins (Hostettman and Nakanishi 1979), terpenoids and flavonoids (Leven et al. 1979) have also been investigated for their antimicrobial

Table 4 Antifungal activity of extracts of A. scholaris and M. pinnata

\begin{tabular}{|c|c|c|c|c|c|c|c|}
\hline \multirow[t]{3}{*}{ Extracts } & \multirow[t]{3}{*}{ Conc. in $\mu \mathrm{g} /$ disc } & \multicolumn{6}{|c|}{ Zone of inhibition (mm) } \\
\hline & & \multicolumn{3}{|c|}{ Alstonia scholaris } & \multicolumn{3}{|c|}{ Millettia pinnata } \\
\hline & & A. alternata & A. solani & F. oxysporum & A. alternata & A. solani & F. oxysporum \\
\hline \multirow[t]{3}{*}{ Aqueous } & 10 & $12 \pm 0.9 \mathrm{e}$ & $-\mathrm{h}$ & $-\mathrm{g}$ & $13 \pm 1.4 \mathrm{i}$ & $9 \pm 0.4 \mathrm{f}$ & $-\mathrm{h}$ \\
\hline & 20 & $19 \pm 1.2 \mathrm{c}$ & $13 \pm 0.3 \mathrm{e}$ & $12 \pm 0.6 \mathrm{e}$ & $20 \pm 0.7 f$ & $16 \pm 0.2 \mathrm{e}$ & $-\mathrm{h}$ \\
\hline & 30 & $28 \pm 1.5 b$ & $19 \pm 0.5 \mathrm{c}$ & $17 \pm 0.5 \mathrm{c}$ & $29 \pm 1.5 \mathrm{c}$ & $27 \pm 0.6 b$ & $11 \pm 0.4 \mathrm{e}$ \\
\hline \multirow[t]{3}{*}{ Ethanol } & 10 & $-\mathrm{g}$ & $-\mathrm{h}$ & $-\mathrm{g}$ & $7 \pm 0.6 \mathrm{k}$ & $-\mathrm{g}$ & $-\mathrm{h}$ \\
\hline & 20 & $-\mathrm{g}$ & $9 \pm 1.1 \mathrm{fg}$ & $-\mathrm{g}$ & $12 \pm 0.6 \mathrm{i}$ & $-\mathrm{g}$ & $-\mathrm{h}$ \\
\hline & 30 & $13 \pm 0.4 \mathrm{e}$ & $16 \pm 0.4 \mathrm{~d}$ & $15 \pm 0.8 \mathrm{~d}$ & $18 \pm 0.4 \mathrm{~g}$ & $9 \pm 0.2$ & $10 \pm 1.4 \mathrm{ef}$ \\
\hline \multirow[t]{3}{*}{ Methanol } & 10 & $8 \pm 0.9 \mathrm{f}$ & $6 \pm 0.4 d$ & $7 \pm 0.7$ & $10 \pm 0.8 \mathrm{j}$ & $9 \pm 1.4 \mathrm{f}$ & $6 \pm 0.6 \mathrm{~g}$ \\
\hline & 20 & $17 \pm 0.5 \mathrm{~d}$ & $10 \pm 0.6 f$ & $12 \pm 1.2 \mathrm{e}$ & $16 \pm 0.5 \mathrm{~h}$ & $17 \pm 1.1 \mathrm{e}$ & $11 \pm 1.7 \mathrm{de}$ \\
\hline & 30 & $26 \pm 1.2 b$ & $17 \pm 0.8 \mathrm{~d}$ & $20 \pm 0.8 b$ & $25 \pm 1.3 \mathrm{~d}$ & $26 \pm 0.8 b c$ & $16 \pm 0.7 b$ \\
\hline \multirow[t]{3}{*}{ n-Hexane } & 10 & $-\mathrm{g}$ & $-\mathrm{h}$ & $-\mathrm{g}$ & -1 & $8 \pm 0.7 \mathrm{f}$ & $-\mathrm{h}$ \\
\hline & 20 & $13 \pm 0.2 \mathrm{e}$ & $-\mathrm{h}$ & $10 \pm 0.7 \mathrm{f}$ & -1 & $15 \pm 1.5 \mathrm{e}$ & $9 \pm 0.4 \mathrm{f}$ \\
\hline & 30 & $20 \pm 0.7 \mathrm{c}$ & $17 \pm 0.9 \mathrm{~d}$ & $16 \pm 0.7 \mathrm{~cd}$ & $30 \pm 0.4 \mathrm{c}$ & $25 \pm 0.4 \mathrm{c}$ & $14 \pm 0.6 \mathrm{c}$ \\
\hline \multirow[t]{3}{*}{ Chloroform } & 10 & $-\mathrm{g}$ & $8 \pm 1.1 \mathrm{~g}$ & $-\mathrm{g}$ & $12 \pm 0.7 \mathrm{i}$ & $-\mathrm{g}$ & $-\mathrm{h}$ \\
\hline & 20 & $8 \pm 1.2 \mathrm{f}$ & $16 \pm 1.2 \mathrm{~d}$ & $11 \pm 0.8 \mathrm{ef}$ & $22 \pm 0.5 \mathrm{e}$ & $15 \pm 1.4 \mathrm{e}$ & $7 \pm 0.9 \mathrm{~g}$ \\
\hline & 30 & $13 \pm 1.1 \mathrm{e}$ & $23 \pm 1.5 b$ & $17 \pm 0.5 \mathrm{c}$ & $32 \pm 1.2 \mathrm{~b}$ & $22 \pm 1.0 \mathrm{~d}$ & $12 \pm 0.4 \mathrm{~d}$ \\
\hline Nystatin & 10 & $31 \pm 1.0 \mathrm{a}$ & $29 \pm 0.9 \mathrm{a}$ & $31 \pm 0.4 \mathrm{a}$ & $34 \pm 0.2 \mathrm{a}$ & $33 \pm 0.8 \mathrm{a}$ & $30 \pm 0.7 \mathrm{a}$ \\
\hline
\end{tabular}

Values were expressed as Mean \pm S.E. Data were statistically analysed through DMRT at $p \leq 0.05$. DMRT was performed using statistical package DSAASTAT (Onofri, Italy) and significance was mentioned using alphabetic letters following each value. Values followed by similar letters showed no significant difference. Values followed by single consecutive letters showed significant difference; whereas, values followed by more than one letter showed no significant difference 
properties. This study indicates groups of metabolites extracted through different solvents, and it will help researchers for isolation of specific biochemical groups from plant material.

In this investigation we used the strains of fungi and bacteria of both gram positive and gram negative genera. They are important microorganisms due to their pathogenicity towards plants and humans. Methanol extract of leaves of both plant species comprised more antimicrobial properties as compared to other solvents (Ahmad et al. 2014). Therefore, methanol can be used to extract antimicrobial material from plants but cannot be encouraged for isolation of specific biochemical groups. The present study also justifies the use of phytochemicals against plant pathogenic fungi. All of the test fungal strains were susceptible to leaf extracts of both plant species. Based on all these results, use of leaf extracts of both these plant species as natural antibacterial agents is justified.

\section{References}

Aboaba OO, Smith SI, Olude FO (2006) Antibacterial effect of edible plant extract on Escherichia coli 0157:H7. Pak J Nutr 5(4):325-327

Ahmad A, Shafique S, Shafique S, Akram W (2014) Penicillium oxalicum directed systemic resistance in tomato against Alternaria alternata. Acta Physiol Plant. doi:10.1007/s11738-014-1500-5

Akram W, Anjum T, Ali B, Ahmad A (2013) Screening of native bacillus strains to induce systemic resistance in tomato plants against fusarium wilt in split root system and its field applications. Int J Agric Biol 15:1289-1294

Azu NC, Onyeagba RA (2007) Antimicrobial properties of extracts of Allium cepa (onions) and Zingiber officinale (ginger) on Escherichia coli, and Bacillus subtilis. Internet J Trop Med 3(2):277-286

Bajwa R, Javaid A, Shafique S, Javaid A, Jabeen K, Shafique S (2008) Fungistatic activity of aqueous and organic solvent extracts of three rice varieties against phytophathogenic fungi. Allelopathy J 22(2):363-370
Bashir Z, Shafique S, Ahmad A, Anjum T, Shafique S, Akram W (2013) Quantification of cellulose contents by transmission spectra of plant tissues. Cellul Chem Technol 47(5-6):509-513

Baswa M, Rath CC, Dash SK, Mishra RK (2001) Antibacterial activity of Karanj (Pongamia pinnata) and Neem (Azadirachta indica) seed oil: a preliminary report. Microbios 105:183-189

Bouamama H, Noel T, Villard J, Benharref A, Jana M (2006) Antimicrobial activities of the leaf extracts of two Moroccan Cistus L. species. J Ethnopharmacol 104:104-107

Brain KR, Turner TD (1975) The practical evaluation of phytopharmaceuticals, Wright-science technical, Bristol Britain 56-64

Damintoti K, Aly S, Antonella C, Saydou Y, Carla M, Jacques S, Vittorio C, Alfred ST (2005) Antibacterial activity of alkaloids from Sida acuta. Afr J Biotechnol 4(12):1452-1457

Hostettman K, Nakanishi K (1979) Moronic acid, a simple triterpenoid keto acid with antimicrobial activity isolated from Ozoroa mucroanta. J Med Plant Res 31:358-366

Hussain A, Zaman MK, Ramteke AM (2010) Antibacterial activity of trunk bark of Alstonia scholaris. Asian J Pharm Clin Res 3(4):45-47

Leven M, VandenBerghe DA, Mertens F, Vlictinck A, Lammens E (1979) Screening of higher plants for biological activities I. antimicrobial activity. Plant Med 36(4):311-321

Sabir IN, Fraser JA, Killeen MJ, Grace AA, Huang CL (2007) The contribution of refractoriness to arrhythmic substrate in hypokalemic Langendorff-perfused murine hearts. Pflugers Arch 454(2): 209-222

Scalbert A (1991) Antimicrobial properties of tannins. Phytochemistry 30:3875-3883

Shafique S, Bajwa R, Shafique S, Akhtar N, Hanif S (2011) Fungitoxic activity of aqueous and organic solvent extracts of Tagetes erectus on phytopathogenic fungus Ascochyta Rabiei. Pak J Bot 43(1): $59-64$

Shafique S, Shafique S, Ahmed A (2013) Ecofriendly response of citrus peels to alternaria leaf spots of tomato: exclusive role of peel phenolics. Int J Agric Biol 15:1236-1242

Sutton S (2011) Measurement of microbial cells by optical density. J Validation Technol 17(1):46-49

Valgas C, de Souza SM, Smânia EFA, Smânia A Jr (2007) Screening methods to determine antibacterial activity of natural products. Braz J Microbiol 38:369-380 